\title{
Charge Redistribution Mechanisms of Ceria Reduction
}

Olle Hellman, N V Skorodumova and Sergey Simak

\section{Linköping University Post Print}

N.B.: When citing this work, cite the original article.

Original Publication:

Olle Hellman, N V Skorodumova and Sergey Simak, Charge Redistribution Mechanisms of Ceria Reduction, 2012, Physical Review Letters, (108), 13, 135504.

http://dx.doi.org/10.1103/PhysRevLett.108.135504

Copyright: American Physical Society http://www.aps.org/

Postprint available at: Linköping University Electronic Press

http://urn.kb.se/resolve?urn=urn:nbn:se:liu:diva-76952 


\title{
Charge Redistribution Mechanisms of Ceria Reduction
}

\author{
O. Hellman, ${ }^{1}$ N. V. Skorodumova, ${ }^{2,3}$ and S. I. Simak ${ }^{1}$ \\ ${ }^{1}$ Department of Physics, Chemistry and Biology (IFM), Linköping University, SE-581 83, Linköping, Sweden \\ ${ }^{2}$ Applied Materials Physics, Department of Materials Science and Engineering, Royal Institute of Technology, \\ SE-100 44 Stockholm, Sweden \\ ${ }^{3}$ Division of Materials Theory, Department of Physics and Astronomy, Uppsala University, Box 516, S-751 20 Uppsala, Sweden
}

(Received 4 October 2011; revised manuscript received 20 January 2012; published 30 March 2012)

\begin{abstract}
Charge redistribution at low oxygen vacancy concentrations in ceria have been studied in the framework of the density functional theory. We propose a model to approach the dilute limit using the results of supercell calculations. It allows one to reproduce the characteristic experimentally observed behavior of composition versus oxygen pressure dependency. We show that in the dilute limit the charge redistribution is likely to be driven by a mechanism different from the one involving electron localization on cerium atoms. We demonstrate that it can involve charge localization on light element impurities.
\end{abstract}

DOI: 10.1103/PhysRevLett.108.135504

PACS numbers: 61.72.Bb, 61.72.Cc, 71.27.+a

Ceria is a core material of many modern environmentally benign technologies [1], which rely on its excellent redox properties. The efficiency of the oxygen vacancy creation and annihilation in ceria can be controlled by external parameters, such as oxygen pressure and temperature, and modified by appropriate doping. To date, a considerable number of experimental [1-5] and theoretical $[1,6,7]$ studies of ceria reduction have been performed. It is known that in stoichiometric $\mathrm{CeO}_{2}$ each cerium atom donates four electrons to fill up the $p$ orbitals of oxygen. When an oxygen vacancy is formed the two electrons, previously occupying the $p$ orbitals of the departed oxygen, are left in the crystal. This extra charge can redistribute in a number of possible ways. It can, for example, stay localized around the vacancy, forming a truly charged vacancy as it happens in typically ionic $\mathrm{MgO}$ [8]. It can also localize on cerium atoms or at some structural defects like impurities, grain boundaries, and interfaces. For pure ceria it has been shown that for concentrations, directly accessible by calculations $\left(\mathrm{CeO}_{2-x}, x>0.03\right.$, i.e., at concentrations of oxygen vacancies exceeding 1.5 at.\% [9], the oxygen vacancy formation is accompanied by the localization of the two electrons on two nearby $\mathrm{Ce}$ atoms, changing their oxidation state from $4+$ to $3+[10,11]$ [see the corresponding density of states (DOS) with a characteristic occupied Ce $4 f$ peak in the band gap in Fig. 1(a)]. In addition, the possibility for a charged vacancy to exist in bulk ceria has theoretically been investigated applying the formalism developed for semiconductors [6]. Despite these studies the question about the charge redistribution in ceria for concentrations not directly accessible in calculations remains open.

The experimentally measured dependences of cerium oxide composition on external oxygen pressure at different temperatures can give us a clue about the physics behind the redox process [2]. The curves have different slopes for very low concentrations $(x<0.005)$, intermediate concentrations $(0.005<x<0.16)$, and higher concentrations, where, according to the $\mathrm{Ce}-\mathrm{O}$ phase diagram, ordered phases appear [1]. These differences suggest that the initial formation of vacancies up to $\mathrm{CeO}_{2-x}$ with $x \sim 0.005$ might go along a mechanism different from the one dominating at higher concentrations.

Although $a b$ initio methods allow one to study high defect concentrations, the dilute limit remains practically inaccessible for direct calculations due to their prohibiting computational cost. Generally, the finite size of the supercell in the DFT calculations is a serious limitation. The calculated properties are affected by the defect-defect image interaction, which should be negligible in the dilute limit. Even the largest supercells ( $\sim 1000$ atoms), currently possible to handle in first-principles calculations, correspond to rather high defect concentrations. To rectify the finite size problem of DFT calculations a formalism using a set of corrections has recently been developed for

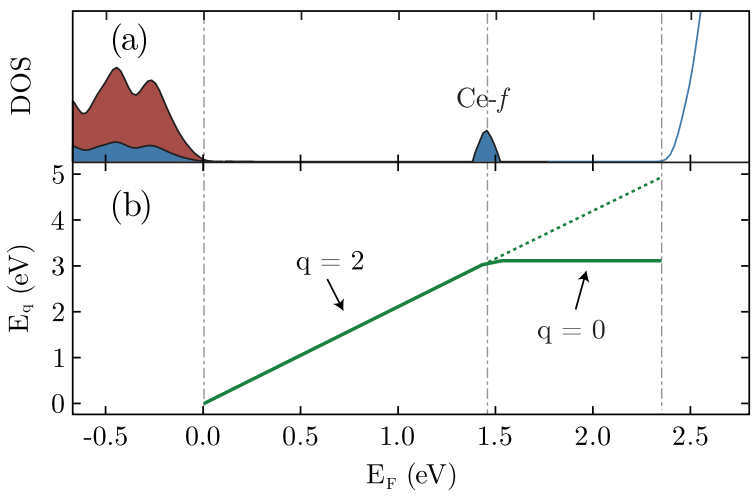

FIG. 1 (color online). (a) Electronic DOS of $\mathrm{CeO}_{2-x}$ showing the electronic structure in the band gap. (b) Plot of $E_{q}\left(E_{F}\right)$ [see Eq. (4)] that is the term in the vacancy formation energy that depends on the Fermi level. The dashed continuation of the line shows what one would expect if the electrons where not allowed to localize on $\mathrm{Ce}$ atoms. 
semiconductors and successfully applied to a number of materials [12].

In this Letter we suggest a method to access dilute vacancy concentrations in oxides, like ceria, focusing on the vacancy formation at the very start of the reduction process in ceria. We show that at the dilute limit the charge redistribution is likely to be driven by a mechanism different from the one involving electron localization on cerium atoms. Our approach allows us to reproduce the characteristic kink in the composition versus oxygen pressure curves observed in experiment [2]. If the standard formalism is applied without consideration of the mixed valence character, as done in Jiang et al. [6], the kink does not appear. When an oxygen vacancy is created two electrons localize on $\mathrm{Ce}$ and change its valence from $4+$ to $3+$. If this is not taken into account, the wrong charged state is assumed. Adding or removing electrons will not have the intended effect of allowing one to study the stability of differently charged states but will cause unphysical strain while not contributing to a better description.

Since we have concluded that the vacancy is formally neutral, adding or subtracting electrons to the supercell does not help in describing the redox behavior of Ce. We suggest another way to model the charge redistribution in ceria, namely, by shifting the Fermi energy in the band gap in accordance with the vacancy concentration. We want to describe the full range of concentrations, corresponding to the Fermi level situated anywhere in the gap between the valence band maximum (VBM) and the conduction band minimum (CBM). Since supercells representing the dilute limit are too big to be studied, we manually unoccupy states above the VBM in a supercell of reasonable size to allow for those electrons to end up in states somewhere in the gap. Accordingly we correct the total energy by

$$
\Delta E_{\mathrm{uo}}=-\int_{\epsilon_{\mathrm{VBM}}}^{\infty} \omega(\epsilon) \epsilon f\left(\epsilon, E_{F}\right) d \epsilon
$$

where subscript uo refers to the unoccupation procedure, $\epsilon_{\mathrm{VBM}}$ corresponds to the energy of the valence band maximum, which is here set to zero with all other energies being shifted accordingly, $f\left(\epsilon, E_{F}\right)$ to the Fermi-Dirac distribution function, and $\omega(\epsilon)$ represents the DOS in the band gap. The energy contribution $\Delta E_{\mathrm{uo}}$ is not a correction per $s e$, but a means to implement our model in the spirit of band-filling correction techniques of the semiconductor formalism used to approach the dilute limit $[12,13]$. If we allow for the proposed charge density redistribution there is no need to add or remove electrons to investigate different charge-reconfigured states. We can model them by positioning the Fermi level in the gap, as it has been done by Janotti et al. [14]. The key difference with the semiconductor approach is that changes in the oxidation state of cerium atoms (and, thus, their formal charge) will be allowed for certain positions of the Fermi level. Therefore, one can divide the redox process into two parts, the early part with a dilute concentration of vacancies and the high-concentration part where electron localization on cerium atoms is the dominant mechanism.

To apply this approach to ceria we have carried out the spin-polarized DFT calculations for the mentioned above $2 \times 2 \times 2$ flourite supercell (96 atoms) with one oxygen atom removed. The projector augmented wave [15] method has been used as implemented in VASP[16-19]. The Brillouin zone integration is done with a $3 \times 3 \times 3$ Monkhorst-Pack [20] mesh using the tetrahedron method according to Blöchl [21], and an energy cutoff for plane waves is set to $400 \mathrm{eV}$. We have used the local density approximation (LDA) [22] for exchange and correlations and applied LDA $+\mathrm{U}[23]$ to treat the strongly correlated $f$ electrons, with $U=6 \mathrm{eV}$ [24]. The excess electrons were localized on two Ce ions closest to the vacancy and the system was allowed to relax until the forces on each ion were less than $0.02 \mathrm{eV} / \AA$. We note that electron localization involving any two atoms in the supercell results in the appearance of a filled $f$ peak in the band gap. Therefore, a consideration of exact $\mathrm{Ce}^{3+}$-vacancy configurations is of little relevance for the present study. We notice that the particular magnetic configuration of the $\mathrm{Ce}^{3+}$ ions is also of minor importance for the present study, so the ferromagnetic ordering is assumed. The charge-image electrostatic interaction in defect calculations is usually corrected for by Makov-Payne corrections [25]. The vacancy formation in ceria creates a charge neutral complex, consisting of two $\mathrm{Ce}^{3+}$ and a formally $2+$ vacancy. Multipole-multipole interaction of such a defect complex with its images is not especially long-ranged. That, together with a high dielectric constant $(\sim 20)$ of the ceria matrix, justifies our choice not to apply Makov-Payne corrections to ceria.

We calculate the vacancy formation energy according to:

$$
E_{v}^{f}\left(x, E_{F}\right)=E_{\left[\mathrm{CeO}_{2-x}\right]}-E_{\left[\mathrm{CeO}_{2}\right]}+x \mu \mathrm{O}+E_{q}\left(E_{F}\right) .
$$

Here $x$ is double the concentration of oxygen vacancies on the oxygen sublattice $(x=2 c) . E_{\left[\mathrm{CeO}_{2-x}\right]}$ is the total energy of the defect supercell. $E_{\left[\mathrm{CeO}_{2}\right]}$ is the total energy of the stoichiometric cell. $\mu_{o}$ is the chemical potential of oxygen. Notice that the last term of Eq. (2), where $q$ is the charge state and $E_{F}$ the Fermi energy, is different from the $q E_{F}$ used for semiconductors. (see, e.g., Ref. [12]). In the case of ceria, the formal charge is a function of the Fermi energy as it changes when electrons localize on Ce $4 f$ states and looks as follows:

$$
E_{q}\left(E_{F}\right)=E_{F} q\left(E_{F}\right)+\int_{\epsilon_{\mathrm{VBM}}}^{\infty} \omega(\epsilon) \epsilon f\left(\epsilon, E_{F}\right) d \epsilon,
$$

where again $\epsilon_{\mathrm{VBM}}$ is set to zero as in Eq. (1). According to Eq. (3), the electrons are allowed to occupy states in the gap, if there are any. Notice that this is the formal charge reconfiguration described by Eq. (1) above. The behavior of function $E_{q}\left(E_{F}\right)$ with changing $E_{F}$ is shown in Fig. 1. When $E_{F}$ has increased above the energy of Ce $4 f$ states in 
this rigid-band model there is no reason why electrons would occupy gap states higher in energy than the Ce $f$ states, except due to temperature smearing of the step in the Fermi-Dirac function. The latter effect is, however, rather negligible for the temperature range of practical interest, so the energy remains basically constant. We stress that energy dependence of $E_{q}\left(E_{F}\right)$ is a physically correct description for ceria. Direct application of the semiconductor formalism, where this energy dependence is omitted, leads to an inadequate treatment of defects in ceria as it has been demonstrated by Jiang et al. [6]. In particular, the results of Jiang et al. [6] clearly show that the crossover of the formation energies of presumably differently charged vacancies, obtained in the semiconductor formalism, takes place at Fermi energies where the $f$ electrons should already be localized. Furthermore, following the standard treatment, we can define the chemical potential of oxygen $\mu_{O}$, as

$$
\mu_{O}=\mu_{O}^{0}+\frac{1}{2} k_{B} T \ln \left(P_{O_{2}}\right),
$$

where $\mu_{O}^{0}$ is the molecular binding energy of oxygen gas, which can be calculated or taken from experiment, to correct for the overbinding of DFT calculations [26]. Setting the lower limit for $\mu_{O}$ to the value of the oxygen chemical potential in bulk ceria and the higher limit to that of the gas, a large partial oxygen pressure interval can be covered [27]. For chosen pressure and temperature, we can estimate the vacancy concentration by

$$
c \approx \exp \left(-\frac{E_{v}^{f}}{k_{B} T}\right)
$$

On the other hand, the number of electrons occupying states in the gap per Ce atom is given by

$$
\frac{n_{e^{-}}^{\text {gap }}}{n_{\mathrm{Ce}}}=\frac{c}{4}=\int_{\epsilon_{\mathrm{VBM}}}^{\epsilon_{\mathrm{CBM}}} \frac{\omega(\epsilon) d \epsilon}{1+\exp \left[\left(\epsilon-E_{F}\right) / k_{B} T\right]}
$$

that can be translated into $E_{F}$ knowing the concentration (one electron per $\mathrm{Ce}$ in the gap corresponds to $\mathrm{Ce}_{2} \mathrm{O}_{3}$, $c=0.25$, provided the $\omega(\epsilon)$ is properly normalized). Thus, solving Eqs. (2), (5), and (6) self-consistently and, taking into account the variation of chemical potential of oxygen with pressure [Eq. (4)], one can obtain a vacancy concentration versus oxygen pressure dependence. This is measured experimentally. The results of this procedure for different temperatures together with the corresponding experimental data are shown in Fig. 2. The calculated curves show a distinctive kink where the mechanism changes to $\mathrm{Ce}$ $f$ localization. Even though the calculated kink is shifted to higher concentrations, the slopes of the theoretical curves at low concentrations agree well with those from experiment. The concentration at which the theoretical kink occurs depends on the distance between the VBM and the Ce $4 f$ peak in the gap. The vertical position of the curves is affected by the errors in estimated $\mu_{O}$, choice of parameter $U$, and exchange-correlation functional. It is also well known that the band gaps calculated in DFT always contain errors with
Concentration of oxygen vacancies, theoretical

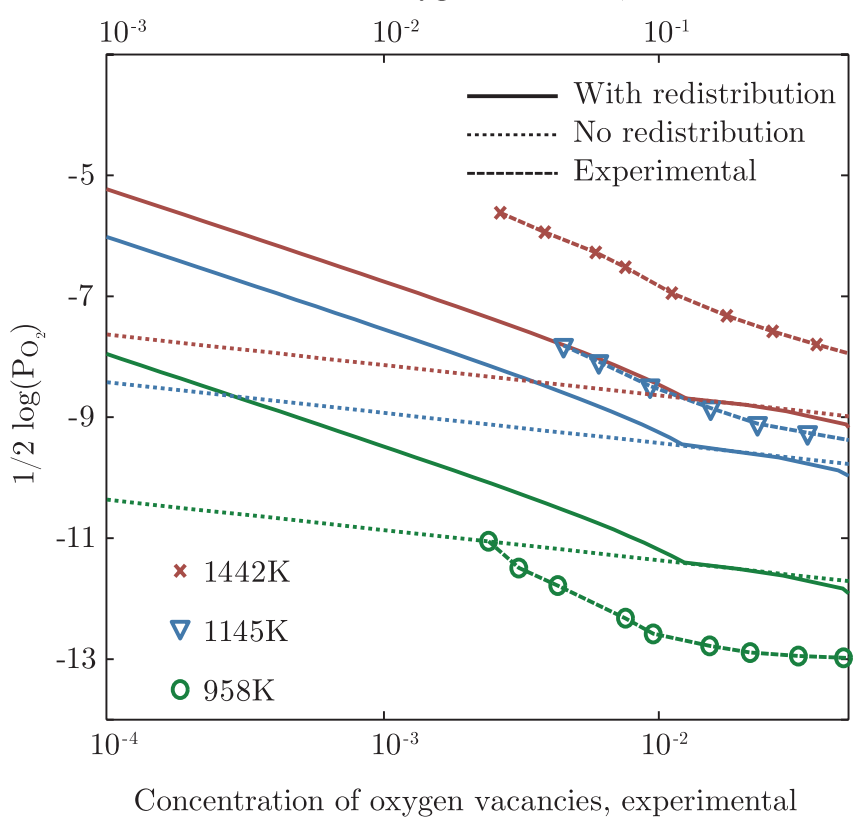

FIG. 2 (color online). The partial oxygen pressure needed to achieve a certain concentration of oxygen vacancies. Solid lines are this work, dotted lines are theoretical values not considering charge density redistribution. The dashed lines are experimental values [2].

respect to the experimental ones. It turns out, however, that to be able to correct for that is not crucial. Most important, the slope only depends on just whether one allows for charge redistribution or not. We note that a detailed description of ordered phases of high vacancy concentrations was not intended in this work. Figure 2 also displays the pressurecomposition dependences when no charge redistribution is allowed, i.e., when formation of $\mathrm{Ce} 3+$ occurs at all concentrations. The asymptotic behavior of the curves clearly shows that this is not an appropriate model in the dilute limit.

The physical conclusion one can draw from the simple model described above is that at the very beginning of the reduction process charge redistribution can be different from localization at Ce $f$ orbitals. Instead, charge can be trapped by impurities, dopants, extended structural defects or interfaces. For higher vacancy concentrations the amount of "hiding places" for electrons would decrease with the increasing number of vacancies and eventually the formation of the $\mathrm{Ce} 3+$ and vacancy complexes would prevail. The model essentially captures the asymptotic behavior and transition between the two concentration regimes (Fig. 2).

To see if light element impurities, common in most materials, can offer an alternative charge redistribution path we have studied nitrogen and carbon impurities in ceria. We notice that $\mathrm{N}$ and $\mathrm{C}$ are the elements whose ions require one and two more electrons to fill their valence $p$ orbitals as compared with $\mathrm{O}$, respectively. In Fig. 3 the densities of states for supercells containing $\mathrm{N}$ $\left(\mathrm{CeN}_{y} \mathrm{O}_{2-x-y}\right)$ and $\mathrm{C}\left(\mathrm{CeC}_{y} \mathrm{O}_{2-x-y}\right)$ impurities and one 


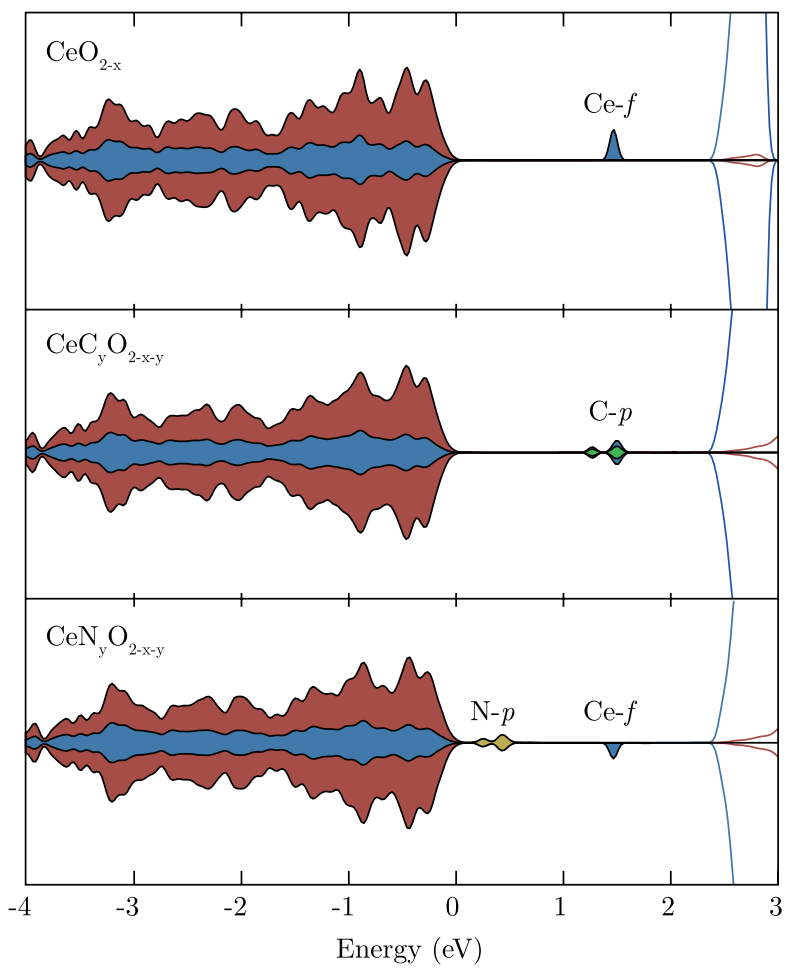

FIG. 3 (color online). Electronic density of states for partially reduced ceria. Top is ceria with no impurities, middle is ceria with a carbon atom occupying an oxygen site and bottom has a nitrogen atom on an oxygen site. In all panels the upper half represents spin up and the lower half spin down DOS.

oxygen vacancy are presented. The calculations show that, in the case of $\mathrm{C}$, both excess electrons end up at the $\mathrm{C}$ orbitals, the absence of occupied $\mathrm{Ce} f$ states indicates that there is no $\mathrm{Ce} 3+$ in the system. In the case of nitrogen, one electron localizes on $\mathrm{N}$, whereas the other one goes to $\mathrm{Ce} f$ orbitals. Such a charge redistribution leads to easier vacancy formation with calculated formation energies, which are lower than that in pure ceria by $0.677 \mathrm{eV}$ and $1.107 \mathrm{eV}$ for $\mathrm{CeC}_{y} \mathrm{O}_{2-x-y}$ and $\mathrm{CeN}_{y} \mathrm{O}_{2-x-y}$, respectively. Although the considered concentration of impurities is rather high, our results clearly demonstrate that there exist common impurities which can change the charge distribution pattern allowing for atomiclike states (Fig. 3). If they are situated below the $\mathrm{Ce} 4 f$ peak they facilitate the formation of oxygen vacancies by absorbing electrons bound for the $f$ states. Additionally, our previous results have shown that some dopants can exhibit similar behavior, in particular, a vacancy formation in $\mathrm{Pb}$-doped ceria is accompanied by electron localization on $\mathrm{Pb}$ orbitals instead of those of $\mathrm{Ce}$, that decreases the vacancy formation energy by $\sim 0.5 \mathrm{eV}$ [28].

To summarize, our results suggest that at the beginning of the redox process the vacancy formation can go along a different mechanism providing lower vacancy formation energies than later in the process. Natural impurities are able to act as catalysts jump-starting the process yielding the redox properties of ceria.
We would like to thank the Swedish Research Council, (VR) for financial support and the Swedish National Infrastructure for Computing (SNIC) for computational resources. The support from the Swedish Government Strategic Research Area Grant in Materials Science including Functional Materials is acknowledged.

[1] A. Trovarelli, in Catalysis by Ceria and Related Materials (Imperial College Press, London, 2002).

[2] D. Bevan, J. Inorg. Nucl. Chem. 26, 1509 (1964).

[3] Z. Shao and S.M. Haile, Nature (London) 431, 170 (2004).

[4] S. Tsunekawa, K. Ishikawa, Z.-Q. Li, Y. Kawazoe, and A. Kasuya, Phys. Rev. Lett. 85, 3440 (2000).

[5] H. Nörenberg and G. Briggs, Phys. Rev. Lett. 79, 4222 (1997).

[6] Y. Jiang, J. B. Adams, M. van Schilfgaarde, R. Sharma, and P. A. Crozier, Appl. Phys. Lett. 87, 141917 (2005).

[7] D. A. Andersson, S. I. Simak, N. V. Skorodumova, I. A. Abrikosov, and B. Johansson, Phys. Rev. B 76, 174119 (2007).

[8] H.-J. Freund and G. Pacchioni, Chem. Soc. Rev. 37, 2224 (2008).

[9] 1.5 at. \% of oxygen vacancies correspond to a 96 atom $2 \times$ $2 \times 2$ ceria supercell with one oxygen atom removed.

[10] A. E. Hughes, J. D. Gorman, P. J. K. Patterson, and R. Carter, Surf. Interface Anal. 24, 634 (1996).

[11] N. V. Skorodumova, S. I. Simak, B. I. Lundqvist, I. A. Abrikosov, and B. Johansson, Phys. Rev. Lett. 89, 166601 (2002).

[12] S. Lany and A. Zunger, Phys. Rev. B 78, 235104 (2008).

[13] T. S. Moss, Proc. Phys. Soc. London Sect. B 67, 775 (1954).

[14] A. Janotti, J. B. Varley, P. Rinke, N. Umezawa, G. Kresse, and C. G. Van De Walle, Phys. Rev. B 81, 85212 (2010).

[15] P. E. Blöchl, Phys. Rev. B 50, 17953 (1994).

[16] G. Kresse and D. Joubert, Phys. Rev. B 59, 1758 (1999).

[17] G. Kresse, Comput. Mater. Sci. 6, 15 (1996).

[18] G. Kresse and J. Furthmüller, Phys. Rev. B 54, 11169 (1996).

[19] G. Kresse and J. Hafner, Phys. Rev. B 48, 13115 (1993).

[20] H. Monkhorst and J. Pack, Phys. Rev. B 13, 5188 (1976).

[21] P. E. Blochl, Phys. Rev. B 50, 17953 (1994).

[22] D. M. Ceperley and B. J. Alder, Phys. Rev. Lett. 45, 566 (1980).

[23] S. L. Dudarev, G. A. Botton, S. Y. Savrasov, C. J. Humphreys, and A.P. Sutton, Phys. Rev. B 57, 1505 (1998).

[24] D. A. Andersson, S. I. Simak, B. Johansson, I. A. Abrikosov, and N. V. Skorodumova, Phys. Rev. B 75, 035109 (2007).

[25] G. Makov and M. Payne, Phys. Rev. B 51, 4014 (1995).

[26] M. Nolan, S. Parker, and G. Watson, Surf. Sci. 595, 223 (2005).

[27] Y. Jiang, J. B. Adams, and M. van Schilfgaarde, J. Chem. Phys. 123, 064701 (2005).

[28] D. A. Andersson, S. I. Simak, N. V. Skorodumova, I. A. Abrikosov, and B. Johansson, Phys. Rev. B 76, 174119 (2007). 